\title{
Oesophageal pH monitoring is of limited value in the diagnosis of "reflux-cough"
}

\author{
R.N. Patterson*,\#, B.T. Johnston*,\#, J. MacMahon*, , L.G. Heaney*, , L.P.A. McGarvey*,
}

Oesophageal pH monitoring is of limited value in the diagnosis of "reflux-cough". R.N. Patterson, B.T. Johnston, J. MacMahon, L.G. Heaney, L.P.A. McGarvey. (C) ERS Journals Ltd 2004.

ABSTRACT: Reflux-cough is a diagnosis based on demonstrating both gastrooesophageal reflux and a positive response to anti-reflux therapy. The authors sought to determine early and long-term response to therapy in patients with a "positive" $24 \mathrm{~h}$ oesophageal pH study, and identify any features which might predict response.

Patients with chronic cough were recruited from July 1998 to July 2002. Those with a positive $\mathrm{pH}$ study were given dietary advice and an 8-week trial of omeprazole $(20 \mathrm{mg}$ b.i.d.). Response was judged after 8 weeks (clinical follow-up), and at long-term followup (telephone questionnaire).

A total of 146 patients underwent pH monitoring with $82(56.2 \%)$ "positive" studies. Follow-up data was available in 60 patients, with 49 receiving anti-reflux therapy, of which $20(40.8 \%)$ reported a positive treatment response. At long-term follow-up (median 30 months), there was a significantly lower response (14 out of 49, 28.5\%), with no significant difference in either acid exposure times (distal/proximal) or symptom correlation between responders and nonresponders at early or long-term follow-up.

In conclusion, despite "positive" pH studies, over half of the patients $(55.1 \%)$ failed to respond to therapy. No features on $\mathrm{pH}$ monitoring accurately predicted response. Short-term response did not predict long-term response. The precise role for $\mathrm{pH}$ monitoring in the assessment of chronic cough remains to be defined.

Eur Respir J 2004; 24: 724-727.
*Dept of Medicine, Queen's University Belfast, ${ }^{\#}$ GI Physiology Unit, Royal Victoria Hospital, and Regional Respiratory Centre, Belfast City Hospital, Belfast, Northern Ireland, UK.

Correspondence: N. Patterson

Dept of Medicine

Mulhouse Building

Royal Victoria Hospital

Grosvenor Road

Belfast

Northern Ireland

BT12 6BJ

UK

Fax: 442890329899

E-mail: neil@rnpatterson.idps.co.uk

Keywords: Anti-reflux therapy

cough

gastro-oesophageal reflux

oesophageal $\mathrm{pH}$ monitoring

Received: January 202004

Accepted after revision: July 162004
Gastro-oesophageal reflux disease (GORD) and chronic cough both occur commonly in the general population $[1,2]$. This has led to the suggestion of a "cause and effect" relationship between the two, although no such association has been firmly established. The consistent finding from clinics that employ diagnostic protocols for the evaluation of chronic cough has been that GORD causes or contributes to the cough in $10-31 \%$ of cases $[3,4]$.

Despite the frequency with which GORD is identified, there is no agreement as to the most effective way to investigate a patient with suspected GORD-related cough. There are no features on history, such as the cough character, or its timing that accurately determine GORD as the cause, and, in $75 \%$ of cases, typical reflux symptoms may be absent $[3,5]$. Ambulatory 24-h oesophageal $\mathrm{pH}$ monitoring has an established role in the diagnosis of GORD in patients with reflux symptoms, and in those with atypical reflux symptoms, such as chest pain. It has had increasing importance in the evaluation of chronic cough, allowing quantification of both the amount of acid reflux and also the temporal association between cough episodes and reflux events [6].

The current authors' experience with a diagnostic protocol, which included oesophageal $\mathrm{pH}$ monitoring in a series of consecutive patients referred to a specialist cough clinic, has previously been reported [7]. A high $(100 \%)$ negative predictive value was found for this test [7]. This study also demonstrated lower positive predictive values $(68 \%)$ for the investigation based on short-term response to anti-reflux therapy. In contrast, other groups have reported much higher positive predictive values ranging $83-91 \%$ [3, 4, 8, 9]. Generally, these findings have been based solely on review of the shortterm response to therapy. There have been no long-term follow-up studies determining the utility of 24-h oesophageal $\mathrm{pH}$ monitoring in identifying treatment responders.

The current authors believe that ordering 24-h oesophageal $\mathrm{pH}$ monitoring in every patient referred for evaluation of cough has practical limitations. In order to determine the clinical value of the investigation, a follow-up study of a new series of cough patients was undertaken with the following objectives: 1) to determine response to anti-reflux therapy in those with positive $\mathrm{pH}$ studies both after an initial trial of therapy and after long-term follow-up; and 2) to identify any features on $\mathrm{pH}$ monitoring (e.g. acid exposure times and/or reflux-cough symptom correlation) that might predict response to therapy.

\section{Methods}

\section{Protocol for cough evaluation}

All patients had been referred between July 1998 and July 2002 to the Regional Respiratory Centre at the Belfast City Hospital, Belfast, Northern Ireland, UK, with a nonproductive cough persisting for $>3$ weeks. During this period, there were 328 patients investigated for specialist assessment of chronic cough. All were aged $18-86 \mathrm{yrs}$, had no previous 
history of chest disease and no upper respiratory tract infection in the preceding 8 weeks. Current smokers or those taking angiotensin-converting enzyme inhibitors were excluded. A comprehensive diagnostic and therapeutic protocol combining diagnostic testing and trials of empirical therapy was followed, based on the approach previously described [7]. In brief, this involved a systematic evaluation of potential causes of cough. After history and physical examination, baseline investigations including chest radiograph and spirometry were arranged in all patients to identify any primary pulmonary cause for the cough. Based on the history and clinical examination, 24-h oesophageal $\mathrm{pH}$ monitoring, bronchoprovocation challenge testing, and, where appropriate, formal ear, nose and throat (ENT) assessment and/or a computed tomography scan of the sinuses were requested. Using this approach, $35(10.7 \%)$ patients were diagnosed with gastro-oesophageal refluxassociated cough based on a trial of empirical anti-reflux therapy alone. Ethics approval was obtained from the Queen's University Belfast and all patients gave informed consent.

\section{pH monitoring}

Ambulatory $\mathrm{pH}$ monitoring was performed over a 24-h period. A monocrystalline antimony electrode (Zinetics 24 ME; Synectics Medical Ltd, Middlesex, UK) was calibrated in buffers of $\mathrm{pH} 7$ and $\mathrm{pH} \mathrm{1,} \mathrm{and} \mathrm{positioned} 5 \mathrm{~cm}$ above the manometrically determined upper border of the lower oesophageal sphincter. The proximal probe was positioned $10 \mathrm{~cm}$ above the distal probe. Any suggestion of abnormal oesophageal motility was followed up by full manometric studies (as follows). Patients documented their cough symptoms both in a diary card and by pressing an event marker on the digitrapper. Data were analysed using the Esophogram program (Gastrosoft Inc. Version 2.04; Gastrosoft, Irving, TX, USA).

Studies were interpreted according to methods used in the current authors' laboratory and those adopted in a previous protocol [7]. Oesophageal $\mathrm{pH}$ monitoring measures the duration of time that the oesophageal mucosa is exposed to acidic conditions $(\mathrm{pH}<4)$. Normal values have been established [10]. Based on these normal values, the present study divided the population of cough patients into those with and without excessive acid reflux. A study was deemed positive if either of the following were present: increased distal oesophageal acid exposure ( $\%$ total time $\mathrm{pH}<4:>4.9 \%, \%$ upright $>6.1 \%, \%$ supine $>1.2 \%$ ) or a positive symptom index ( $\geqslant 33.3 \%$ of coughs occurring within $2 \mathrm{~min}$ (before and/or after) of a fall in $\mathrm{pH}$ to $<4$ at the distal probe).

Patients with either increased acid exposure or positive symptom index received advice regarding lifestyle measures (weight reduction, posture, diet) and a trial of omeprazole (20 mg b.i.d), a proton pump inhibitor (PPI), for 8 weeks. At follow-up, a diagnosis of reflux-cough was made in those who responded to therapy. Category of response is detailed as follows.

\section{Oesophageal manometry}

Oesophageal manometry was performed using a standard $4.5 \mathrm{~mm}$ diameter, water-perfused, six-channel oesophageal manometry catheter (Dentsleeve Esophageal LES sleeve catheter; Medtronics, Minneapolis, MN, USA). The catheter was passed transnasally and positioned with the most distal channel in the stomach, the next most proximal (Dent sleeve; Medtronics) in the lower oesophageal sphincter, and the remaining channels in the oesophagus at $5,10,15$ and $20 \mathrm{~cm}$ above the lower oesophageal sphincter. With the patient supine, five "wet" swallows, each consisting of $5 \mathrm{~mL}$ of water, were given at 20-s intervals. Peristaltic activity and lower oesophageal sphincter function were observed, and, if any abnormalities were detected, a further five 5-mL "wet" swallows were given ("full" manometric study). On completion of the swallows, the catheter was slowly withdrawn, the upper oesophageal sphincter position and function observed, and the catheter removed.

\section{Review strategy}

Response was judged at a routine clinic review after 6-8 weeks, following a trial of anti-reflux therapy and at longterm follow-up by telephone interview. Patients were categorised according to their response to anti-reflux therapy as follows: 1) complete, if cough symptoms had completely resolved; 2) partial, if there had been an improvement in cough symptoms without complete resolution; and 3) no response, if symptoms persisted as previously.

\section{Statistics}

Acid exposure times (normal values for the current authors' unit: $\%$ time $\mathrm{pH}$ total $<4.9 \%, \%$ upright $<6.1 \%, \%$ supine $<1.2 \%$ ) at both the proximal and distal probe were compared between responder and nonresponder groups. Response rate was compared between short- and long-term follow-up using Chi-squared analysis. Statistical analysis of the data was performed using nonparametric Mann-Whitney U-test.

\section{Results}

A total of 146 patients from the Regional Cough Clinic (Regional Respiratory Centre, Belfast City Hospital) underwent oesophageal $\mathrm{pH}$ monitoring for chronic cough during the study period. A total of $82(56.2 \%)$ had positive $\mathrm{pH}$ studies. Chart review and telephone questionnaires were completed in $60(73.2 \%$; 41 female, mean age $57.1 \mathrm{yrs}$, range 25-86 yrs; tables 1 and 2), of whom 49 received a trial of antireflux therapy.

\section{Early follow-up}

Chart review of the 49 patients $(81.7 \%)$ who received antireflux therapy indicated that $20(40.8 \%)$ had either partial or complete resolution of cough and $27(55.1 \%)$ had no response. In two patients, no record of response was documented in the notes at review.

Table 1.-Acid exposure times and correlation on oesophageal $\mathrm{pH}$ monitoring

\begin{tabular}{lcc}
\hline$\%$ time & Responder & Nonresponder \\
\hline Subjects n & 20 & 27 \\
Distal total & $7.2(4.1-13.0)$ & $5.5(3-6.3)$ \\
$\quad$ Upright & $10.7(4.0-16.4)$ & $6.2(3.3-9.5)$ \\
$\quad$ Supine & $2.3(0.4-7.9)$ & $1.2(0.1-9.7)$ \\
Proximal total & $0.7(0.2-5.3)$ & $0.5(0.3-0.85)$ \\
$\quad$ Upright & $1.0(0.3-8.4)$ & $0.6(0.05-0.85)$ \\
$\quad$ Supine & $0.0(0.0-0.3)$ & $0.0(0.0-0.7)$ \\
Reflux cough correlation \% & $33.3(4.2-64.6)$ & $25(0.0-44.4)$ \\
\hline
\end{tabular}

Data are presented as $\mathrm{n}$ or median (interquartile range). There was no significant difference found between responder and nonresponder groups (early follow-up). 
Table 2. - Acid exposure times and correlation on oesophageal $\mathrm{pH}$ monitoring

\begin{tabular}{lcc}
\hline$\%$ time & Responder & Nonresponder \\
\hline Subjects n & 14 & 35 \\
Distal total & $7.6(4.5-14)$ & $5.5(3.2-7.9)$ \\
$\quad$ Upright & $11.6(8.0-20.4)$ & $6.2(3.2-9.5)$ \\
$\quad$ Supine & $2.9(0.3-7.0)$ & $1.7(0.1-9.7)$ \\
Proximal total & $0.2(0.1-8)$ & $0.5(0.35-1.0)$ \\
$\quad$ Upright & $0.3(0.1-12.5)$ & $0.8(0.3-0.95)$ \\
$\quad$ Supine & $0.0(0.0-0.1)$ & $0.0(0.0-0.65)$ \\
Reflux cough correlation \% & $38.9(12.5-57.4)$ & $33.3(0.0-46.2)$ \\
\hline
\end{tabular}

Data are presented as $\mathrm{n}$ or median (interquartile range). There was no significant difference found between responder and nonresponder groups (long-term follow-up).

A total of $11(18.3 \%)$ patients did not receive anti-reflux therapy despite the positive study, either because of spontaneous resolution of cough $(n=6)$, or because they received treatment for an alternative diagnosis as per the authors' protocol $(n=4)$, or because of failure to receive prescribed treatment $(n=1)$. This group of untreated patients did not significantly differ from the treated patients in any demographic or oesophageal $\mathrm{pH}$ parameter.

\section{Long-term follow-up}

Follow-up by telephone interview was performed a median of 30 months (range 7-51 months) after initiation of therapy. Of the 49 patients who received a trial of therapy (including the two patients whose early response was not documented in the notes), $14(28.5 \%)$ had either a complete or partial response and $35(71.4 \%)$ remained symptomatic despite extensive additional investigation and trials of therapy.

Long-term follow-up of the 11 patients who did not receive anti-reflux therapy confirmed six cases $(54.5 \%)$ in whom the cough had resolved completely, four patients remained on alternative therapies based on alternate positive test results and diagnoses, and cough persisted in the one patient who failed to receive prescribed treatment. A total of $10(50 \%)$ patients who initially responded to anti-reflux therapy had a recurrence of their cough (fig. 1).

\section{Oesophageal pH data}

There was no difference in either acid exposure times or reflux-cough correlation between responders and nonresponders. This was true for both the early and long-term response (tables 1 and 2). No abnormalities of oesophageal motility were detected in any of the groups.

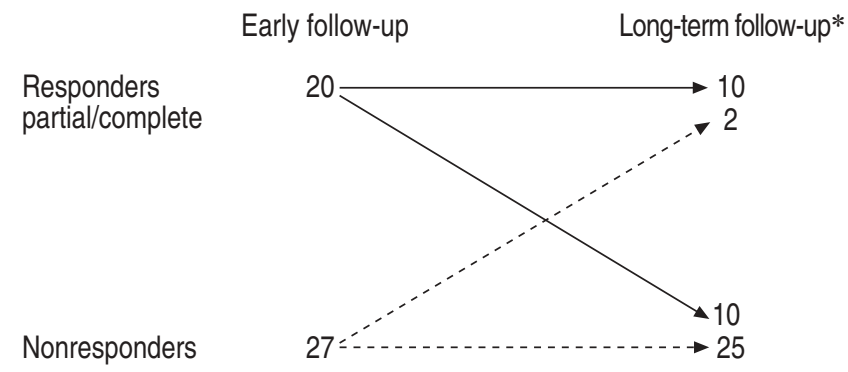

Fig. 1.-Comparison of numbers of early and long-term response to anti-reflux therapy for responders (-) and nonresponders (----). Data are presented as $\mathrm{n} .{ }^{*}: \mathrm{p}<0.05$ versus the early response.

\section{Anti-reflux medication}

Of the 27 patients who did not have an initial response to therapy, 15 patients $(56 \%)$ were still taking their PPI at longterm follow-up, despite no improvement in cough (10 of these were on the basis of GORD symptoms). In the group who did not respond, compliance with long-term anti-reflux therapy, was assessed using a prescription count, a recognised surrogate for treatment compliance. Primary care physicians were contacted, and all patients who claimed they were still taking antireflux therapy were collecting regular repeat prescriptions.

\section{Smoking}

During the telephone follow-up of patients, seven individuals admitted that they were current smokers. Four of the seven patients were in the responder group and three were nonresponders at early follow-up (two of these three had resolution of their cough at long-term follow-up).

\section{Discussion}

The principle finding of the current study is that 24-h oesophageal $\mathrm{pH}$ monitoring has a poor positive predictive value for both short- and long-term response to anti-reflux therapy among coughers with proven GORD. This finding must be qualified by our policy of selecting for $\mathrm{pH}$ monitoring only those who either had no GORD symptoms or who had no improvement in cough after an empirical trial of PPI therapy. Although this was a selected population, the present authors believe that meaningful conclusions can be drawn, as the approach that this study adopted reflects current practice, avoiding the need for an invasive test in patients who respond to a simple trial of therapy [11].

A second important lesson to draw from this study is the difference in outcome when measured at 8 weeks and after 2 yrs. Half of the patients deemed to have responded to PPI therapy at early review regarded themselves as nonresponders at 2 yrs (fig. 1). Potential reasons for this difference include an early placebo response or relaxation of compliance with initial lifestyle modification advice (dietary and smoking) at later follow-up. Whatever the reason, it does highlight the need to interpret with caution any study that only reports short-term response to therapy. It also emphasises the need for long-term follow-up studies in this difficult area.

In this cohort, $56 \%$ of cough patients considered suitable for $\mathrm{pH}$ monitoring had a positive study and over half of these individuals $(55 \%)$ failed to respond to anti-reflux therapy. Neither acid exposure times at distal or proximal probes nor reflux cough correlation accurately predicted response to antireflux therapy. These findings are at variance with much of the existing literature that has commended the utility of $\mathrm{pH}$ monitoring in the evaluation of cough. In one study by VAEZI and RICHTER [8], 91\% of patients with a positive pH study required only modest doses of anti-reflux therapy to completely resolve cough symptoms. However, a later report from the same group found a response rate of only $35 \%$ in patients testing positive by ambulatory $\mathrm{pH}$ monitoring in a randomised controlled trial of omeprazole [12]. The findings from the present observational study are consistent with those published in such randomised controlled trials and contrast with response rates reported in many other prospective studies. It is believed that this supports the current conclusions of only modest responses to anti-reflux therapy.

Possible reasons why half of the studied patients failed to respond to anti-reflux therapy could include inadequate dose 
or duration of medication, noncompliance and unidentified co-existent causes for cough. Omeprazole, at a dose of $40 \mathrm{mg}$ q.d., has been shown to suppress acid secretion by $85 \%$ [13]. In a study by POE and KALLAY [14], cough resolution occurred within 6 weeks in $95 \%$ of patients responding to a similar dose of omeprazole. However, a small number of patients with persisting dyspepsia were identified in the present study, perhaps suggesting suboptimal dosing. In previous studies, the current authors have described some patients who continue to have ongoing acid secretion despite being on PPIs and have also reported the occasional need for higher than usual doses of PPI to control acid production and improve coughing $[15,16]$. It is possible that factors other than acid contribute to the triggering of cough. Volume distension of the oesophagus with nonacid refluxate may be of importance in the pathogenesis of cough. Oesophageal impedance measurements can accurately define nonacid intraluminal events. When performed simultaneously with $\mathrm{pH}$ monitoring, multichannel intraluminal impedance has great potential to contribute to the understanding of reflux and its association with cough [17].

It is possible that concurrent diagnoses were overlooked in some cases. However, alternative aetiologies were considered using the authors' cough algorithm. Of the 27 patients who did not have an initial response to anti-reflux therapy, 18 had a histamine challenge test performed with 10 negative and eight positive studies (provocative concentration causing a $20 \%$ fall in the forced expiratory volume in one second, range $\left.1.92-3.93 \mathrm{mg} \cdot \mathrm{mL}^{-1}\right)$. All patients with a positive histamine challenge were maintained on inhaled corticosteroids, but, at follow-up, the cough persisted. Patients with negative bronchoprovocation studies were also given an empirical trial of inhaled steroids in an attempt to identify those with eosinophilic bronchitis [18]. Patients with upper airway symptoms had an ENT assessment and/or a trial of antirhinitic therapy. Therefore, the current authors feel it is unlikely that major aetiologies were missed in those patients still coughing at long-term follow-up.

In a recent paper by KASTELIK et al. [19], a high prevalence of abnormal oesophageal manometry in patients presenting with chronic cough was observed. Whilst full manometric studies were not performed in all these patients, as this was not the aim of the present study, a series of five "wet" swallows, both in the oesophageal body and in the lower oesophageal sphincter, did not reveal any motility abnormalities in this population. This suggests that, in the current population, oesophageal dysmotility is unlikely to play a significant role in reflux-associated cough.

More work is required to identify the most useful oesophageal tests and parameters that can best guide therapy. The addition of simultaneous impedance recording to detect nonacid reflux and the use of a cough monitor to record cough events more accurately would be beneficial.

In summary, over half of cough patients with a positive $\mathrm{pH}$ study failed to respond to anti-reflux therapy. Inadequate dose or duration of therapy, or failure to identify alternative or co-existing diagnoses are unlikely explanations for this. In predicting response to therapy, neither the acid exposure times nor the degree of correlation between reflux events and cough episodes were accurate for either early or late response.

Although this study reflects the current authors' local practice, it is believed that these observations are important for physicians who request and interpret this test as part of the evaluation of patients with persistent cough.

\section{References}

1. Kahrilas PJ. Gastroesophageal reflux disease. JAMA 1996; 276: 983-988.

2. Irwin RS, Boulet L-P, Cloutier MM, et al. Managing cough as a defense mechanism and as a symptom: a consensus panel report of the American College of Chest Physicians. Chest 1998; 114: 2 Suppl. Managing, 133S-181S.

3. Irwin RS, French CL, Curley FJ, et al. Chronic cough due to gastro-oesophageal reflux: clinical, diagnostic and pathogenetic aspects. Chest 1993; 104: 1511-1517.

4. Irwin RS, Curley FJ, French CL. Chronic cough. The spectrum and frequency of causes, key components of the diagnostic evaluation, and outcome of specific therapy. Am Rev Respir Dis 1990; 141: 640-647.

5. Mello CJ, Irwin RS, Curley FJ. Predictive values of the character, timing, and complications of chronic cough in diagnosing its cause. Arch Intern Med 1996; 156: 997-1003.

6. Laukka MA, Cameron AJ, Schei AJ. Gastroesophageal reflux and chronic cough: which comes first? $J$ Clin Gastroenterol 1994; 19: 100-104.

7. McGarvey LPA, Heaney LG, Johnston BT, et al. Evaluation and outcome of patients with chronic non-productive cough using a comprehensive diagnostic protocol. Thorax 1998; 53: 738-743.

8. Vaezi ME, Richter JE. Twenty-four hour ambulatory oesophageal $\mathrm{pH}$ monitoring in the diagnosis of acid refluxrelated chronic cough. South Med J 1997; 90: 305-311.

9. Smyrnious NA, Irwin RS, Curley FJ. Chronic cough with a history of excessive mucus production: the spectrum and frequency of causes, key components of the diagnostic evaluation, and outcome of specific therapy. Chest 1995; 108: 991-997.

10. Johnston BT, McFarland RJ, Collins JS, Love AH. Symptom index as a marker of gastro-oesophageal reflux disease. Br J Surg 1992; 79: 1054-1055.

11. Schnatz PF, Castell JA, Castell DO. Pulmonary symptoms associated with gastroesophageal reflux: use of ambulatory $\mathrm{pH}$ monitoring to diagnose and to direct therapy. Am J Gastroenterol 1996; 91: 1715-1718.

12. Ours TM, Kavuru MD, Richter JE, et al. A prospective evaluation of esophageal testing and a double-blind, randomised study of omeprazole in a diagnostic and therapeutic algorithm for chronic cough. Am J Gastroenterol 1999; 94: 3131-3138.

13. Lind $\mathrm{T}$, Cederberg $\mathrm{C}$, Olausson $\mathrm{M}$, et al. Omeprazole in elderly duodenal ulcer patients: relationship between reduction in gastric acid secretion and fasting plasma gastrin. Eur J Clin Pharmacol 1991; 40: 557-560.

14. Poe RH, Kallay MC. Chronic cough and gastroesophageal reflux disease: experience with specific therapy for diagnosis and treatment. Chest 2003; 123: 679-684.

15. Leite LP, Johnston BT, Just RJ, et al. Persistent acid secretion during omeprazole therapy: a study of gastric acid profiles in patients demonstrating failure of omeprazole therapy. Am J Gastroenterol 1996; 96: 1529-1531.

16. Johnston BT, Gideon RM, Castell DO. Excluding gastroesophageal reflux disease as a cause of chronic cough. $J$ Clin Gastroenterol 1996; 22: 168-169.

17. Castell DO, Vela M. Combined multichannel intraluminal impedance and pH-metry: an evolving technique to measure type and proximal extent of gastroesophageal reflux. $\mathrm{Am}$ J Med 2001; 111: Suppl. 8A, 157S-159S.

18. Brightling $\mathrm{CE}$, Ward $\mathrm{R}$, Goh $\mathrm{KL}$, et al. Eosinophilic bronchitis is an important cause of chronic cough. $\mathrm{Am}$ J Respir Crit Care Med 1999; 160: 406-410.

19. Kastelik JA, Redington AE, Aziz I, et al. Abnormal oesophageal motility in patients with chronic cough. Thorax 2003; 58: 699-702. 